\section{PHILOSOPHICAL TRANSACTIONS B}

royalsocietypublishing.org/journal/rstb

\section{Review}

Cite this article: Peyrache A, Seibt J. 2020

A mechanism for learning with sleep spindles.

Phil. Trans. R. Soc. B 20190230.

http://dx.doi.org/10.1098/rstb.2019.0230

Accepted: 29 December 2019

One contribution of 18 to a Theo Murphy meeting issue 'Memory reactivation: replaying events past, present and future'.

\section{Subject Areas: \\ neuroscience, cognition}

\section{Keywords:}

sleep, spindles, memory, plasticity, coupling, reactivation

\section{Authors for correspondence:}

Adrien Peyrache

e-mail: adrien.peyrache@mcgill.ca

Julie Seibt

e-mail: j.seibt@surrey.ac.uk

\title{
A mechanism for learning with sleep spindles
}

Adrien Peyrache ${ }^{1}$ and Julie Seibt ${ }^{2}$

${ }^{1}$ Montreal Neurological Institute, McGill University, Montreal, Quebec Canada, H3A 1A1

${ }^{2}$ Surrey Sleep Research Centre, University of Surrey, Guildford, UK

AP, 0000-0001-9708-309X; JS, 0000-0002-1081-9680

Spindles are ubiquitous oscillations during non-rapid eye movement (NREM) sleep. A growing body of evidence points to a possible link with learning and memory, and the underlying mechanisms are now starting to be unveiled. Specifically, spindles are associated with increased dendritic activity and high intracellular calcium levels, a situation favourable to plasticity, as well as with control of spiking output by feed-forward inhibition. During spindles, thalamocortical networks become unresponsive to inputs, thus potentially preventing interference between memory-related internal information processing and extrinsic signals. At the system level, spindles are co-modulated with other major NREM oscillations, including hippocampal sharp wave-ripples (SWRs) and neocortical slow waves, both previously shown to be associated with learning and memory. The sequential occurrence of reactivation at the time of SWRs followed by neuronal plasticity-promoting spindles is a possible mechanism to explain NREM sleep-dependent consolidation of memories.

This article is part of the Theo Murphy meeting issue 'Memory reactivation: replaying events past, present and future'.

\section{Introduction}

Soon after the discovery of brain waves by Hans Berger in 1929 [1], Alfred Loomis performed electroencephalographic (EEG) recordings in resting subjects and provided the first descriptions of sleep-specific brain oscillations [2]. The EEG traces during sleep, far from being flat as expected from an 'inactive' brain, are dominated by various patterns, including the so-called spindles, short (less than $3 \mathrm{~s}$ ) waxing and waning oscillatory events with a typical frequency ranging from 9 to $15 \mathrm{~Hz}$ in humans [3-7]. The neuronal basis underlying the generation of spindles has now been clearly established and involves the interplay between the neocortex and thalamic networks spontaneously oscillating at spindle frequency [8-10].

A decade before the discovery of spindles, sleep had been shown to be beneficial for memories [11], and the relationship between sleep dynamics and memory has remained a central topic of neuroscience since then [12-16]. Specifically, a novel experience imprints a labile physiological trace in the brain that vanishes unless it is consolidated. Sleep is considered to be an ideal brain state for reprocessing memories in order to sort, consolidate and generalize recently acquired knowledge $[15,17]$. The exact contribution of different sleep stages for memory consolidation remains a subject of debate [13,18-20], yet it is generally accepted that non-rapid eye movement (NREM) sleep plays a key role in this process $[15,20]$. Oscillations during NREM sleep have been proposed to be the correlates of cellular and molecular mechanisms promoting sleep-dependent memory enhancement [12,13,19-21]. Among the different oscillations observed during NREM sleep, thalamocortical spindles have been repeatedly suggested as a mediator of cognitive enhancement and memory improvement observed during sleep [5,8,22-24].

Sleep spindles have now been the focus of a large body of literature and studied in a variety of conditions: different animal species and brain modalities, natural sleep and anaesthesia, sleep with or without associated learning 
paradigms, in vivo and in vitro. Here, we will first review our current understanding of the link between spindles, memory and synaptic plasticity. Then, we will review the recent data that link spindles and local regulation of the neocortical microcircuitry and how this regulation supports their role in synaptic plasticity. We will then address the question of the role of spindles at the systems level and how their co-modulation with other major oscillations during NREM sleep, in particular hippocampal sharp wave-ripples (SWRs), may constitute the physiological basis of the NREM sleep-dependent consolidation of declarative memories. Finally, we propose a model in which spindles play a central role for synaptic consolidation in the particular framework of memory systems consolidation.

\section{Link between spindles, memory and plasticity}

Being the most prominent sleep stage (approx. 80\% of total sleep in adult humans), NREM sleep has attracted of lot of attention as the main mediator of sleep function(s). While REM sleep has long been a focus of memory research, the discovery of experience-dependent NREM 'replay' activity in the hippocampus provided new evidence and interest in the role of NREM sleep in memory [25-27].

\section{(a) Thalamocortical non-rapid eye movement sleep oscillations}

Cortical activity during NREM sleep differs from wakefulness in one crucial aspect: it is synchronized by slow waves (SWs). SWs are typically divided into slow oscillations (SOs less than $1 \mathrm{~Hz}$ ) and Delta (1-4 Hz), sometimes grouped into slow wave activity (SWA: $0.5-4 \mathrm{~Hz}$ ). In thalamocortical networks, the other NREM-specific oscillations are the spindles. In humans, SWs and spindles do not occur at the same rate across sleep and their relative prevalence divides NREM sleep into three stages indicative of sleep depth [18,28]. Although spindles are found across all NREM sleep stages, spindle activity prevails in stage 2 (light sleep) while SWA increases in stage 3 (deep sleep, also called slow-wave sleep, SWS) [29]. In rodents, the architecture of NREM sleep can also be divided into three stages during which cortical dynamics share strong similarities with human electrophysiological markers defining these stages [18]. Similar to humans, SWs and spindle activity are more prominent during stages $\mathrm{N} 2$ and N3 in rodents but spindle intensity and number show a greater increase at the end of NREM sleep episodes [30-32], which often correspond to the transition to REM sleep.

The physiology of SWs and spindles has now been well characterized and is the focus of several comprehensive reviews [5,7-9,33-36]. These oscillations are both generated within thalamocortical networks. However, while the primary generator of SWs consists of the cortical fluctuation between UP and DOWN states of neuronal activity [9], the pacemaker of spindles resides in the thalamus. Briefly, spindles are generated by the interplay between the inhibitory cells of the thalamic reticular nucleus (TRN) of the thalamus and thalamocortical neurons in the dorsal thalamus [9,36]. Corticothalamic Inhibitory volleys from the reticular cells activate specific calcium channels (T-type current) which eventually leads to bursts of action potentials in some thalamocortical neurons. This strong volley of excitation, in turn, activates the inhibitory cells of the TRN, and this interaction repeats itself at each spindle cycle. These excitatory volleys also depolarize cortical neurons, giving rise to spindle events, as detected in EEG and local field potential (LFP) recordings (figure 1a). While the neocortex does not directly play a role in the generation of spindles, corticothalamic projections synchronize these oscillations [41].

Fine differences in spindle frequencies are observed across developmental stages and brain regions, yet, overall they are preserved across animal species [42]. In humans, spindles are readily visible in the EEG and can be divided into fast $(12-15 \mathrm{~Hz})$ and slow $(9-12 \mathrm{~Hz})$ spindles based on distinct peaks in the NREM power spectra $[43,44]$. The differentiation into fast and slow spindles is also based on topography whereby fast spindles are preferentially expressed in the parietal/somatosensory areas and slow spindles in the frontal brain areas ([43] and see [5] for review). Although this distinction may have some functional relevance in relation to memory in humans (see below), the fast and slow components of spindles cannot be distinguished in rodent EEG/LFP power spectra [44] which limits their study in this species. However, differences in power and occurrence rate have been observed across cortical areas in rodents $[45,46]$. Spindles are not a unitary phenomenon, but instead, reflect the oscillatory activity of many parallel thalamocortical circuits that may or may not synchronize across circuits and modalities. In fact, intracerebral spindles recorded in epileptic patients implanted with depth electrodes are oftentimes 'local' and more rarely 'global' $[43,47,48]$. Furthermore, spindles simultaneously monitored with EEG and magnetoencephalography, believed to track the activity of different cortical dipoles, show only a weak temporal correlation [37].

While the ubiquitous nature of spindles and their characteristics across brain regions and animal species become less and less disputed, it is only in the last 20 years that their contribution to memory and brain plasticity, in general, has started to be unveiled.

\section{(b) Link between spindles and memory}

The strong activation of cortical neurons by thalamic excitatory volleys is an interesting candidate for promoting plasticity during NREM sleep $[49,50]$. Spindles are associated with both declarative and procedural learning (reviewed in [5]), further suggesting a fundamental link with the mechanisms responsible for reshaping network connectivity. The evidence linking SW and spindle oscillations and memory fall into three categories: correlative, causal and link with plasticity. In this section, we will review each of these categories of experimental findings.

\section{(i) Correlative evidence}

Change in NREM oscillations following learning was first evidenced in human studies. Specifically, using scalp EEG recordings, studies showed that specific spindle parameters (e.g. density, amplitude) increased in post-learning sleep for both declarative and procedural memory tasks ([22,23,38, 51-55] and figure 1b). It is noteworthy that not all forms of learning upregulate spindles (e.g. [56]). In addition, modulation of spindles is also use-dependent as learning induces increase in spindles in brain regions involved in the learned tasks $[55,57-60]$. Those latter studies in humans also show a preferential involvement of fast spindles in memory enhancement. Comparable observations have been made for SWs, in particular SWA, that show use-dependent increase during post-learning 


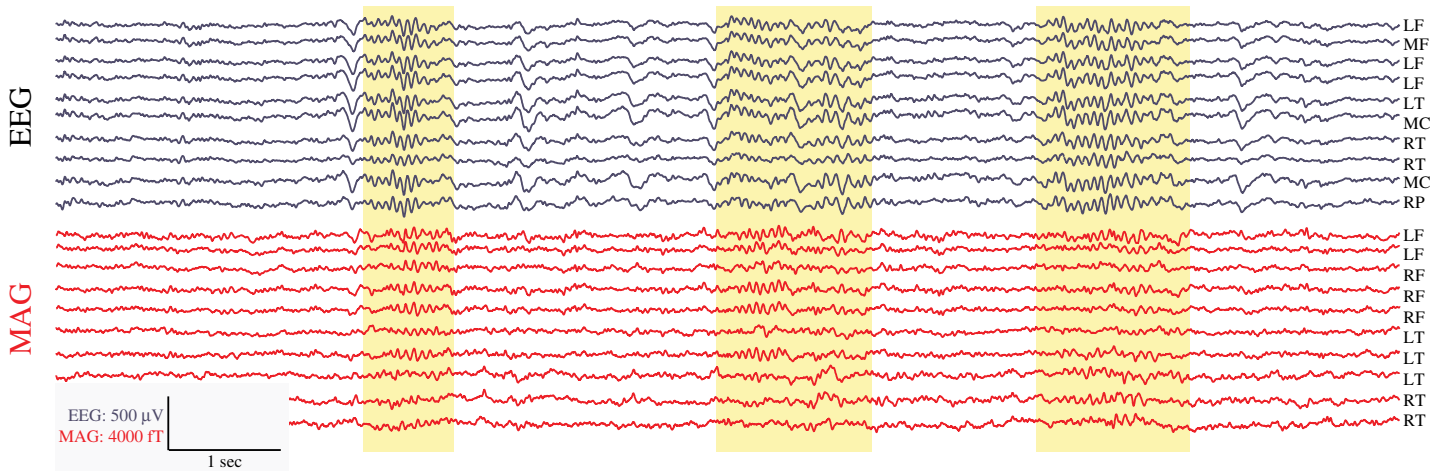

rodent (rat)

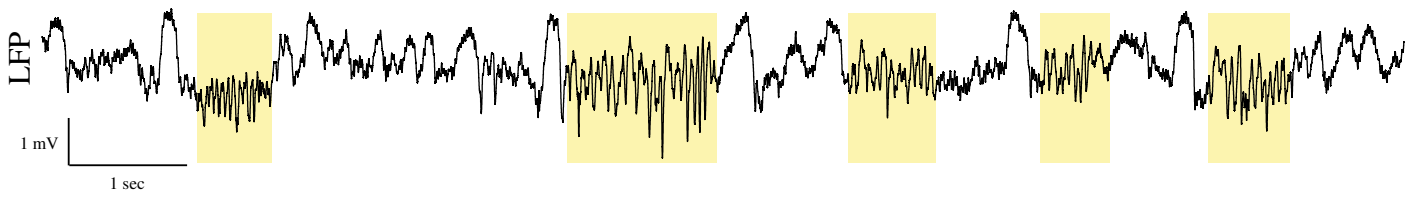

(b)
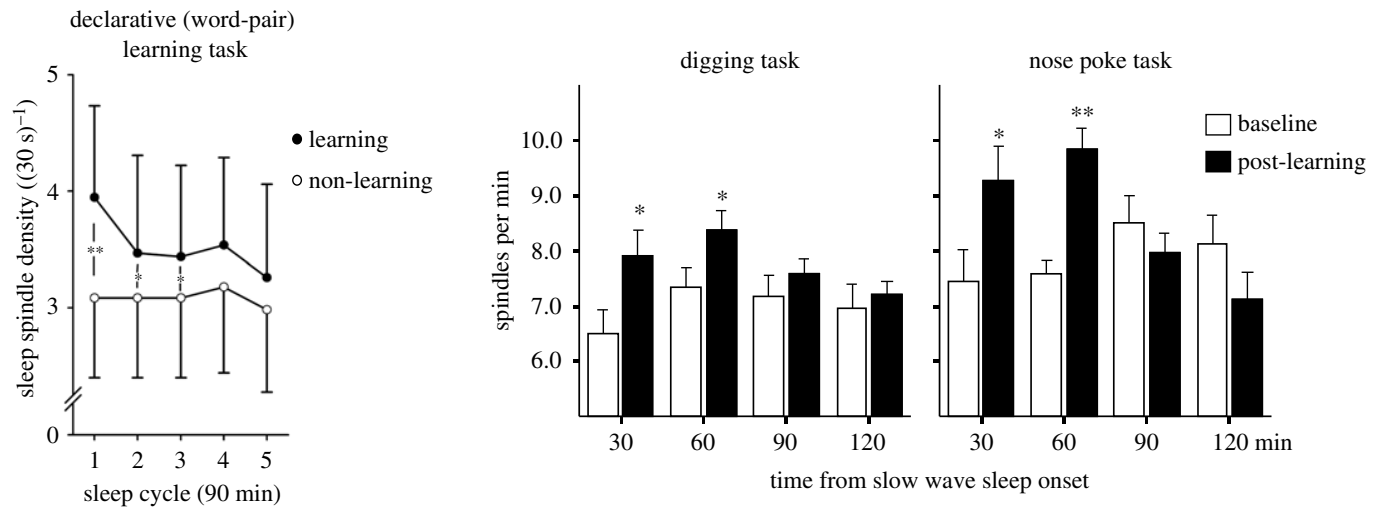

(c)

declarative (word-pair) learning task + so-tDCS $(0.75 \mathrm{~Hz})$ slow frontal spindle activity
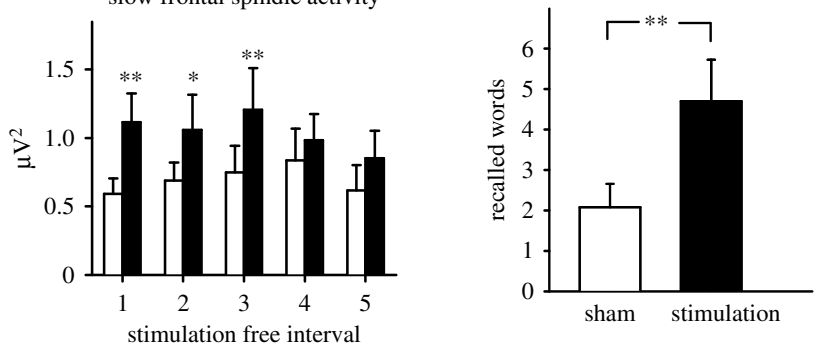

(d)
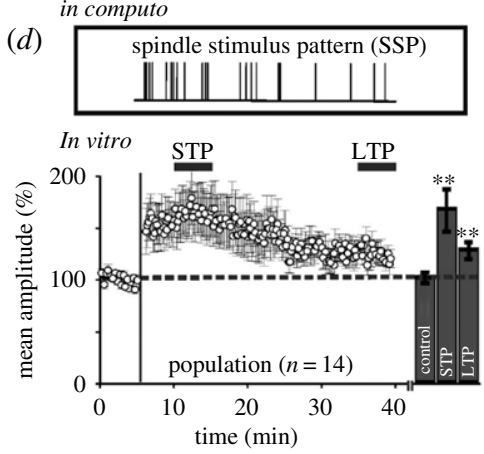

Figure 1. Spindles, memory and plasticity. (a) Examples of spindles in human electroencephalograph (EEG) and magnetoencephalogram (MAG) (upper traces, adapted from [37]) and rat (lower trace, unpublished data from J.S.). Spindles are highlighted in yellow. $(b)$ Influence of learning on spindle density in the Q4 human (left graph, adapted from [38]) and rat (right graphs, adapted from [39]) frontal cortex. (c) Boosting frontal slow spindles (and SOs, not shown) with frontolateral slow oscillatory $(0.75 \mathrm{~Hz}$ ) transcranial direct current stimulation (so-tDCS) in humans improves declarative memory (adapted from [21]). (d) Naturalistic spindle stimulus pattern (SSP) promotes short- (STP) and long-term plasticity (LTP) in vitro (adapted from [40]).

sleep specifically over the cortical areas engaged in the learning task [61,62]. In rodents, both spindles and SWs also increase following learning and experience [39,44,63,64]. Furthermore, during sleep following perceptual visual learning in developing cats and adult mice, neurons of the visual cortex become more strongly phase-locked to spindles than in baseline condition $[65,66]$. Taking together, these data support a functional implication of these oscillations in offline information processing during spindles.

Beyond their role in memory, the relation between spindles and cognition is also supported by studies showing a link between spindles and activity in the developing brain. Evidence points to a role for spindles in the early establishment of sensorimotor cortical maps [67-69]. Furthermore, changes in spindle characteristics are also closely linked to neuropathology associated with cognitive impairments such as autism spectrum disorders, schizophrenia and Alzheimer's disease [70-72]. Although the specific changes in spindles vary depending on the neuropathology (i.e. neuropsychiatric, neurodevelopmental and neurodegenerative disorders) and the spindle characteristics measured, a general trend in decreased spindles density in those pathologies have been repeatedly reported (reviewed in [5]). This suggests that variations in the number of spindles may play a particularly important role for cognitive functions. Taken together, spindles have been cast in variety of roles related to the development 
and maintenance of cognitive functions, including memory. These observations support a common role for spindles in neuronal plasticity - the biological substrate of memory.

\section{(ii) Causal evidence}

Do changes in NREM oscillations, especially spindles, play an active role in memory consolidation or do they only reflect physiological modifications associated with plasticity, such as increased excitability [19]? While correlative studies cannot unequivocally address this issue, it can be addressed via direct manipulations of these oscillations. The best way to establish a direct link between NREM oscillations and cognition is to perform manipulations (e.g. gain- and loss-of-function) of these network phenomena and assess the resultant effect on memory performance. Manipulations of SOs and spindles have been performed in both humans and rodents. In most human studies, non-invasive brain stimulation via transcranial electrical stimulation (tES) is used for this purpose [73]. In a seminal tES study by Marshall and colleagues, they applied transcranial direct current stimulation at the slow oscillation frequency $(0.75 \mathrm{~Hz})$ (so-tDCS) above frontolateral regions in young subjects. This stimulation elicits an increase in both SOs and spindles and improves performance in a declarative paired-associate memory task ([21] and figure 1c). Following this study, these results have been replicated and complemented. Using feedback-controlled transcranial alternating current stimulation (tACS) of frontal areas at spindle frequency $(12 \mathrm{~Hz})$ during NREM sleep, Lustenberger et al. [74] specifically enhanced spindle activity (i.e. sigma $11-16 \mathrm{~Hz}$ ) which was accompanied with enhancement of motor memory consolidation. In addition, increasing the SOs/spindle coupling using administration of zolpidem in humans has a positive effect on performance on a verbal memory task [75]. Targeted memory reactivation (TMR) paradigm is another interesting approach [73]: presentation of a memory-related tone or odour during NREM sleep improves memory performance the following day [76,77]. In addition to enhance memory, TMR elicits a parallel increase in SOs and fast spindle incidence, suggesting a causal relationship with cognitive performance $[21,53,58,78,79]$. Finally, a recent study in humans used bed rocking at slow frequency $(0.25 \mathrm{~Hz})$ to enhance stage 3 NREM $\mathrm{SO}$ and spindles. In this paradigm, memory performance improvement is positively correlated with fast spindles [80]. However, a similar approach in mice shows small effects of rocking on NREM EEG power, in particular, for the sigma band that actually displayed a strong suppression when the frequency of rocking was increased [81]. Although indicative, the influence of rocking on memory should be replicated in mice to clarify the link between NREM oscillations and cognitive performance using this behavioural approach.

In rodents, manipulations of SWs and/or spindle oscillations also point to a relationship with learning and memory. For example, optogenetic stimulation of the TRN to phase lock thalamocortical oscillations (approx. $8 \mathrm{~Hz}$ ) with SOs and hippocampal SWRs boosts the performance of mice in a contextual fear conditioning task [82]. However, it is worth mentioning that this optogenetic stimulation increases spindle oscillations in the 7$10 \mathrm{~Hz}$ frequency band but also inhibits oscillations in the sigma frequency band (10-15 Hz) [83]. Thus, although thalamocortical stimulation during NREM sleep enhances memory in mice, whether the induced oscillations represent physiological spindles remains to be determined. More recently, Kim et al. [84] used optogenetic stimulation in mice to manipulate the amounts of SO/spindle or delta/spindle nesting events and show increased or decreased performance in a neuroprosthetic task (i.e. procedural memory), respectively [84]. This latter study has two implications. First, it supports growing evidence for physiological [45] and functional differences between SO and delta oscillations. Secondly, it further suggests that the role of spindles is not limited to strengthening of memory traces but can, depending on their coupling, also promote forgetting [84]. Altogether, manipulations of NREM sleep oscillations point at an important role of oscillatory coupling in memory, an aspect discussed in $\S 4$.

\section{(iii) Link with plasticity}

Although NREM oscillations may have a direct impact on various aspects of neuronal physiology, their influence on synaptic plasticity is a likely candidate for the mechanisms of memory consolidation. Synaptic plasticity mechanisms, such as long-term potentiation (LTP) and depression (LTD), are thought to be the cellular substrates of long-term modifications of memory-related circuits [85-89]. There are only a handful of studies that have attempted to demonstrate a link between network events during sleep and plasticity measures. In humans, the variability in LTP-like or LTD-like induction using electrical nerve stimulation paired with TMS correlates with inter-individual variability in local changes in slow spindles and the efficacy of the paired stimulation the next day [90]. In mice, Durkin et al. used an in vivo model of cortical plasticity induced by visual experience (orientation-specific response potentiation, OSRP) and showed that optogenetic disruption of corticothalamic activity reduces both spindles and SWA. This manipulation impairs OSRP and the potentiation of neuronal responses in the visual cortex during NREM sleep post-experience [63]. Using a combination of in vivo intracellular and in vitro recordings in the cat neocortex, Chauvette et al. demonstrated that thalamocortical activity during NREM sleep can trigger cortical LTP-like responses [91]. Finally, stimulating in vitro slices of the somatosensory cortex with naturalistic spindle stimuli derived from intracellular recordings in vivo induces both short- term potentiation (STP) and LTP ([40] and figure 1d).

Using a modelling approach, Contreras et al. [92] showed that pyramidal neuron activity during spindles may be particularly beneficial for intracellular calcium $\left(\mathrm{Ca}^{2+}\right)$ increase in dendrites (figure 2a), an important mechanism for induction of synaptic plasticity. Experience-dependent increase in spindles during NREM sleep correlates with increased phosphorylation of $\mathrm{Ca}^{2+} /$ calmodulin-dependent protein kinase II (CaMKII) during REM sleep in the hippocampus [94], suggesting that plasticity initiated during NREM spindles may carry on across sleep stages.

In conclusion, spindles are crucial for the consolidation of several types of memories (i.e. declarative and procedural), as well as for the development of thalamocortical circuits and cortical functional maps. In vitro and in silico studies suggest a common underlying physiological mechanism yet, how neuronal plasticity is induced and regulated during spindles in vivo remains to be determined.

\section{Spindles' role in synaptic plasticity and memory: cellular, local circuits and systems}

While there is now a good understanding of how spindles are generated in the thalamus, the physiology of spindles in the 
(a)
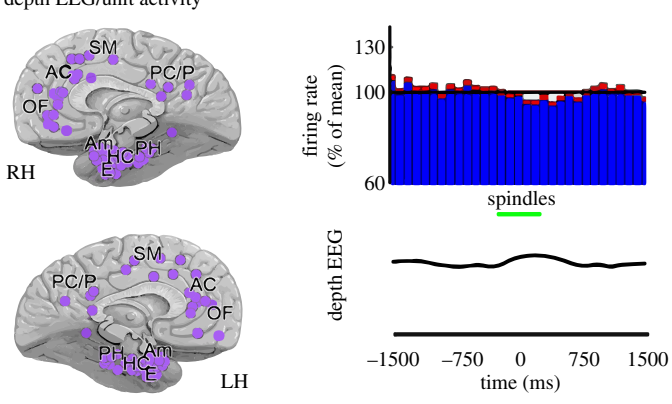

(c)
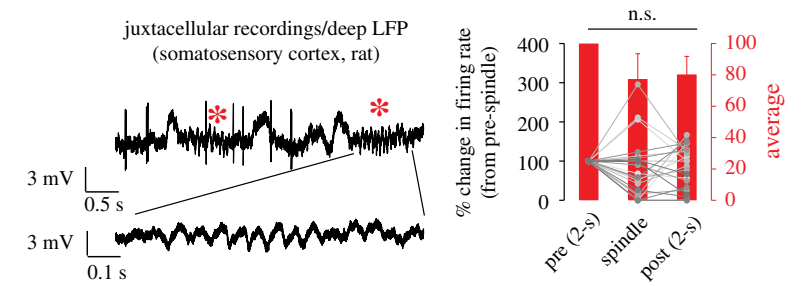

(d)
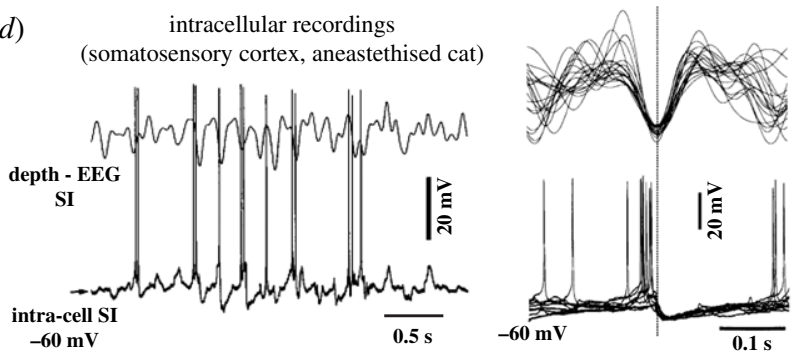

(b)

Figure 2. Inhibition of cortical activity during spindles. (a) Depth EEG/unit activity recordings in various brain regions (purple circles) in humans. Average cortical firing rate (across various depths and brain regions) during spindles (green bar) (adapted from [43]). (b) Entrainment of regular spiking (RS, putative excitatory) and FS (putative inhibitory) neurons during spindles in the superficial and deep layers of rat mPFC. Top trace and raster: example of a spindle event (grey bar) and associated firing in the superficial and deep layers of the $\mathrm{mPFC}$. The red and blue dots in the raster indicate spike times of two putative FS neurons in the superficial (sup.) and deep layers, respectively. Polar histograms show the distribution of the phases of these two neurons relative to all spindles. Bottom left: distribution of preferred phases to spindles for all neurons across layers and cell types (black histogram) and only for the significantly modulated neurons (colour histograms). Bottom right: changes in firing rate during spindles relative to NREM sleep across cell types and layers (adapted from [93]). (c) Average change in firing rate perispindles (red stars) of L5 pyramidal neurons in the somatosensory cortex of head-fixed rats (adapted from [31]). (d) Intracellular recordings of pyramidal neurons activity during spindles in the primary somatosensory cortex (SI) of anaesthetized cats. Left: example of spindles sequence recorded with depth electroencephalogram (depth - EEG) together with neuronal discharge. Right: superimposition of 10 spindle cycles of the same cell. Traces were aligned to negative peaks of depth EEG. Note the EPSP followed by IPSP during spindles (adapted from [92]).

cortex is much less clear. Resolving this question is of prime importance to understand the role of spindles in memory and plasticity. This can only be achieved by a combination of techniques investigating intracellular and network-level properties associated with spindles, and how they relate to memory.

\section{(a) Local: cortical activity during spindles \\ (i) Electrophysiological recordings}

Given that thalamic neurons burst during spindles, cortical neurons must receive strong and synchronized barrage of excitatory inputs that may in turn trigger plasticity in the form of LTP [49] and augmented neuronal responses [50]. However, in vivo electrophysiological recordings in humans and rodents reveal a more complex picture. In humans, cortical neuronal spiking shows little change, if any, during spindles ([43] and figure 2a). While Andrillon et al. [43] studied more than 200 units in various brain regions, the different layers and types of neurons recorded were not specified as determining the precise location of microwires used for intracerebral human electrophysiology is generally a near-impossible task. Using extracellular recordings in naturally sleeping rats, Peyrache et al. [93] showed that spindles entrain most, but not all cortical neurons in the medial prefrontal cortex (mPFC). Specifically, inhibitory neurons across all layers show increased firing rate and phase-locking to spindles. Interestingly, while the majority of superficial excitatory neurons is modulated by spindles, spiking of excitatory neurons in deep layers (Layers 5/6) is indifferent to spindle occurrence and phase ([93] and figure $2 b$ ). The lack of modulation of deep layer excitatory neurons by spindles was confirmed in the somatosensory cortex of head-fixed rats using juxtacellular recording ([31] and figure 2c).

Thalamic inputs to the cortex recruit strong inhibition [95-98], especially by directly activating fast-spiking (FS), putative parvalbumin-positive $(\mathrm{PV}+)$ basket cells $[95,98]$. During spindles, FS cells increase their firing rates and are phase-locked to the oscillations $[93,99]$. Furthermore, the observation that deep layer pyramidal neurons are not modulated by spindles is intriguing as Layer 5 (L5) neurons receive a direct input from thalamocortical neurons [100]. One explanation is 


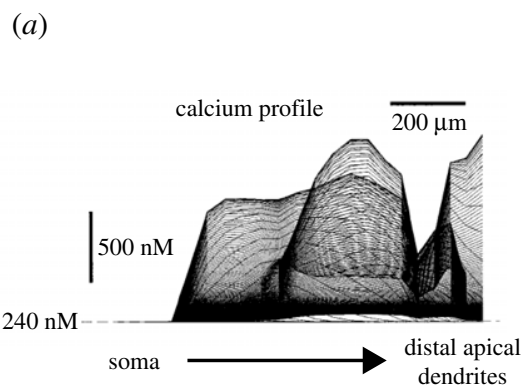

(c) (i)

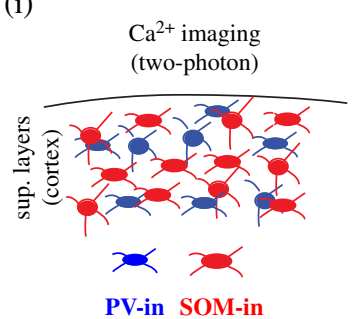

(ii)

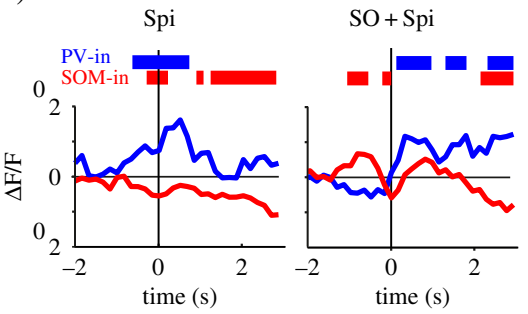

(b)

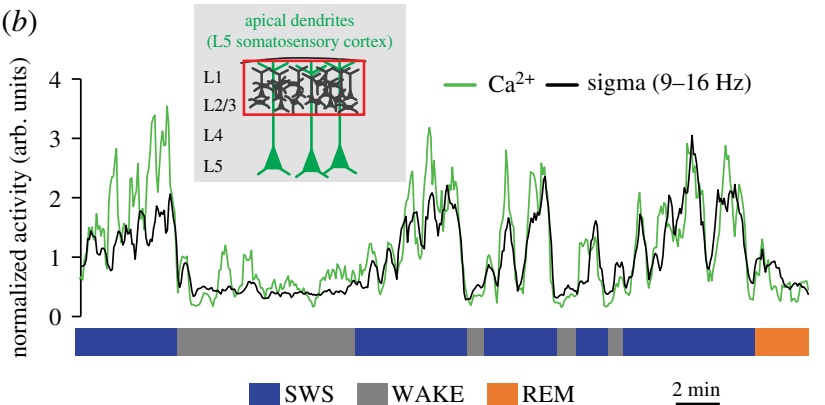

Figure 3. Cortical microcircuit regulation during spindle activity. (a) Model of predicted deep layers (L5/6) somatic and dendritic intracellular Ca ${ }^{2+}$ activity during spindles. Instantaneous profiles taken every $0.2 \mathrm{~ms}$ are superimposed. Note the high $\mathrm{Ca}^{2+}$ activity only in dendrites and not in soma (adapted from [92]). (b) In vivo $\mathrm{Ca}^{2+}$ imaging (photometry) in apical dendrites of $\mathrm{L} 5$ neurons (somatosensory cortex) in freely behaving and sleeping rats. Superimposed activity of population of dendrites and sigma power for an approximately 30 min recording in a freely moving rat (adapted from [31]). (c) (i) In vivo Ca ${ }^{2+}$ imaging (two-photon) of cortical inhibitory neuron subpopulations (PV-in: parvalbumin inhibitory; SOM-in: somatostatin inhibitory) in superficial layers of the cortex. (ii) Mean Ca ${ }^{2+}$ activity aligned to spindles with or without SO coupling. Bars indicate significant differences compared to baseline. (iii) $\mathrm{Ca}^{2+}$ activity changes during and after spindle occurrence (adapted from [101].

that L5 pyramidal neurons, dominated by somatic inhibition, may be indirectly silenced during spindles. This hypothesis is supported by in vivo intracellular recordings in anaesthetized cats showing inhibitory post-synaptic potentials (IPSPs) during spontaneous spindles in the cortex ([92] and figure $2 d$ ). The reason why somatic inhibition of L5 pyramidal neurons is promoted during spindles is not clear, but computational modelling suggests that this could facilitate activation of dendrites without triggering excessive spiking during spindles ([49,92] and figure $3 a$ ). Recent $\mathrm{Ca}^{2+}$ imaging studies have provided evidence that spindles may, in fact, trigger cell- and layer-specific activation in accordance with this model (reviewed in [102,103].

\section{(ii) Calcium imaging}

The advent of optical techniques, such as $\mathrm{Ca}^{2+}$ imaging, has provided new opportunities to monitor brain activity during natural sleep in rodents [103]. Unlike electrophysiology, $\mathrm{Ca}^{2+}$ imaging has the advantage of enabling activity monitoring in selected neuronal populations (e.g. inhibitory subpopulations) and compartments (e.g. dendrites). Imaging of pyramidal and inhibitory neurons at different cortical depths has confirmed the complex regulation of cortical activity during spindles. Using one- and two-photon imaging, Seibt et al. imaged $\mathrm{Ca}^{2+}$ activity in populations and single dendrites of L5 pyramidal neurons in rodents. They found that large populations of dendrites increase their activity and synchronization during spindle-rich oscillations ([31] and figure 3b), supporting the dendritic-centred model proposed by Contreras and colleagues [92]. Using two-photon imaging, Niethard et al. confirmed the electrophysiological findings, suggesting that activity in excitatory and PV+ inhibitory neurons in superficial layers (L2/3) of the cortex correlates with spindle activity ([101] and figure 3c). This latter study further showed that excitatory activity is specifically increased in spindles coupled with SOs while activity in PV+ interneurons is modulated by spindles in general. By contrast, somatostatin positive (SOM+) interneurons in the superficial layers of the cortex show a negative correlation with spindles. Since $\mathrm{SOM}+$ interneurons are known to preferentially target dendrites [104], these results suggest that dendrites are disinhibited during spindles, providing a mechanism underlying increased dendritic activity at the time of spindle occurrence [49,92]. Although imaging approaches suggest that PV+ activity is increased during spindles, this has been characterized only in superficial layers. It remains to be determined if this trend is also found in the deep layers which would support the hypothesis that spindles trigger perisomatic inhibition of L5 neurons.

\section{(b) Systems: coupling of forebrain oscillations during \\ non-rapid eye movement sleep}

One of the best examples of the contribution of spindles to learning is their postulated role in systems consolidation of declarative memories. During NREM sleep, thalamocortical spindles are co-modulated with hippocampal activity, and this coordination is believed to be essential for systems consolidation. Specifically, declarative memories are initially dependent on the integrity of the hippocampus and this dependency gradually declines as memories are consolidated in the neocortex [25,105-109]. During NREM sleep, hippocampal activity is dominated by SWRs, transient events of high neuronal synchronization, associated with a fast $(200 \mathrm{~Hz})$ and short-lasting (50-300 ms) oscillations in the LFP ([110] and figure 4a). SWRs during sleep are critical for memory consolidation $[25,112,113]$ 

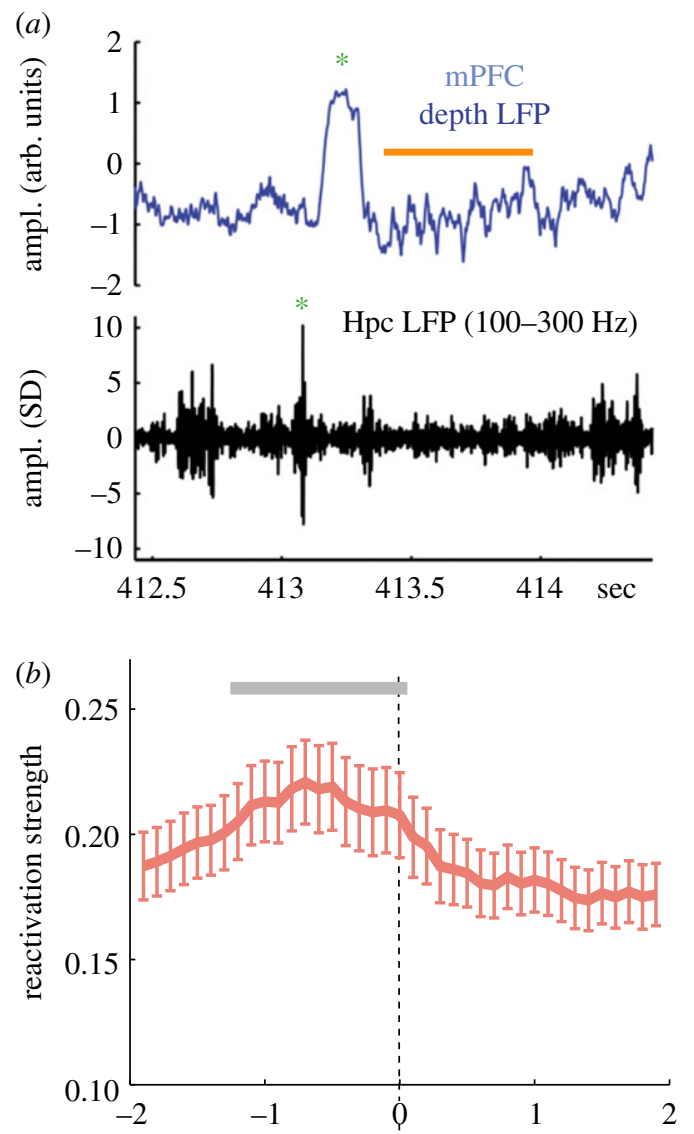

Figure 4. SWR and spindles comodulation and link to replay. (a) Example traces of LFP recorded simultaneously in the medial prefrontal cortex (mPFC, top, broadband) and in the CA1 pyramidal layer of the hippocampus ( $\mathrm{Hpc}$, bottom, filtered in the SWR-frequency range) (adapted from Peyrache et al. [93]. Red and green asterisks indicate a SWR and a SW, respectively. Spindle are highlighted with an orange bar. (b) Relationship between spindles and reactivation in the mPFC. Top: spindle-trough time average $(+/-$ s.e.m.) of reactivation strength of neuronal ensembles during sleep following learning; bottom, same as top for SWR occurrence rate (adapted from [111]).

and the coupling between spindles, SOs and SWRs supports sleep-dependent memory consolidation $[15,114]$.

\section{(i) Hippocampal sharp wave-ripples and the consolidation of declarative memories}

During NREM sleep, patterns of neuronal population activity in the hippocampus that form with experience are spontaneously 'replayed' (or 'reactivated') (for review, see [115,116]), especially at times of SWRs $[27,117]$. This replay of population activity is critical for the consolidation of hippocampus-dependent memories $[25,110]$ as demonstrated by direct manipulation of SWRs $[112,113]$. SWRs are associated with replay of activity in the neocortex, especially in the main output structures of the hippocampus such as entorhinal cortex $[118,119]$ but see [120]), prefrontal cortex [111], striatum [121,122] and amygdala [123]. Interestingly, these brain areas are all well known for their involvement in various forms of learning. As discussed above, learning induces changes in the incidence rate of spindles and SOs (see §2b). Similarly, the occurrence rate of SWRs during NREM sleep increases after learning [124-126]. Overall, these observations suggest that learning is associated with physiological changes (e.g. cell's excitability) that directly affect NREM oscillations characteristics.

\section{(ii) Coupling slow oscillations, spindles and sharp wave-ripples across brain regions}

Declarative memories, especially episodic memories, are supposed to entail broadly distributed networks of neurons across sensory modalities [127]. Large-scale interaction between thalamocortical activity and SWRs during NREM sleep is a possible mechanism that coordinates widely distributed neuronal ensembles to promote memory consolidation $[15,114]$.

The coordination between SWRs and spindles was first evidenced by Siapas \& Wilson [128]. In this seminal study, the authors performed simultaneous recordings of the mPFC as well as CA1 region of the hippocampus and revealed that SWRs tended to occur at the onset of spindle episodes detected in $\operatorname{mPFC}[93,128,129]$ and figure $4 a, b)$. While SWRs and spindles tend to co-occur at slow timescales (approx. $10 \mathrm{~s}$ ) irrespective of the cortical regions where spindles are detected [93,128,130], the sequential ordering of SWRs followed by spindles at faster timescale (approx. $1 \mathrm{~s}$ ) is less pronounced in parietal cortex [130]. This could result from the relative asynchrony of frontal spindles with other brain regions, as observed in rodents [129] and humans [43].

One difficulty in assessing the coordination between SWRs and spindles is that they are both co-modulated with SOs [9,129-132]. Whether the coupling between SWRs and spindles is mediated by cortical inputs to the hippocampus [130] or by a direct communication between the thalamus and the hippocampus remains an open question. Specifically, SWRs may phase-lock to spindles by direct entorhinal projection to CA1, or indirectly via the perforant path through the dentate gyrus and CA3, both areas showing high modulation by spindles [133]. Yet, recent evidence has pointed out a complex coordination between the thalamus and SWRs [134-136], and possibly an active contribution of the thalamus to the timing of SWRs [135]. Although cortical response to SWRs during spindles is strongly diminished [93], hippocampal replay at the time of SWRs is strengthened during spindles [134]. However, the view that SWRs are 'nested' within spindles is not necessarily correct. While SWRs are phase-locked to ongoing spindles [130], only a small proportion of SWRs do occur during spindles, at least in the prefrontal regions [93].

By leveraging the high-channel intra-EEG recordings performed in pre-operative epileptic patients, a growing body of work focuses on the relationship between thalamocortical and hippocampal oscillations. In humans, hippocampal SWRs have recently been associated with memory functions $[137,138]$. Furthermore, SOs, spindles and SWRs are co-modulated, recapitulating results from the rodent literature 
$[139,140]$. As observed in rats, SWRs tend to precede frontal spindles and the delay between SWRs and spindle is less pronounced for parietal spindle [139]. Whether this reflect a 'propagation' of SWR-triggered spindles from frontal to parietal cortex [129] remains an open question. Human studies are still in their infancy but this fast-growing field will make possible to bridge the gap between animal research and human cognitive science.

\section{The sequential hypothesis of hippocampo- cortical coupling for memory}

In the previous sections, we have described (1) how spindles are associated with brain plasticity mechanisms and how they are linked with local cortical activity modulation and (2) how the coupling of spindles with other NREM oscillations promotes different stages of memory formation. In this section, we merge those functions and propose a model whereby spindles are mediators of local synaptic plasticity in the cortex following (and not during) network reactivation of hippocampo-cortical network in the context of declarative memory formation.

\section{(a) The temporal aspect of sharp wave-ripples/spindle coupling}

While SWRs are intimately associated with reactivation of neocortical ensembles, simply 're-activating' neocortical neurons across brain regions may not be sufficient to reinforce mutual coupling between neurons and hence create a stable memory trace. Reactivation may favour a form of tagging of the synapses that are recruited during the reinstatement of the associated neuronal pattern, and the occurrence of spindles following reactivation events is perfectly suited for the synaptic consolidation of these traces. The role of NREM oscillation coupling in sleep-dependent memory consolidation was recently addressed by directly manipulating the temporal relationship between these events. By electrically stimulating the neocortex immediately after SWRs, Maingret and coll. [141] showed that the SO phase is reset, leading to increased incidence of SWR-spindle sequences. Performing this stimulation during sleep following a hippocampus-dependent memory task enhances memory performance the following day [142].

In the $\mathrm{mPFC}$, neuronal reactivation is maximal prior to spindles [111] and this relationship is fully explained by the temporal coordination between SWRs and spindles (figure $4 a, b)$. In this particular case, this observation rules out any specific role of spindles in reactivation, and points instead to a specific coupling between MPFC reactivation and hippocampal SWRs. Unlike other neocortical areas, the MPFC in rats receives direct input from the hippocampus [143] and plays a key role in the systems consolidation of hippocampaldependent memories [144]. Overall, these results suggest that the sequential occurrence of SWRs and frontal spindles is important for the consolidation of hippocampus-dependent memories. According to the proposed hypothesis, synapses that undergo plasticity during spindles ('synaptic consolidation', figure 5a) are part of the neuronal ensembles reinstated during preceding SWRs ('network reactivation', figure 5a). Hence, the role of spindles for hippocampal-dependent memories would be similar to any other form of spindlebased learning. The only difference is that the consolidation of declarative memories in the neocortex additionally requires the hippocampus to reactivate neuronal ensembles before synapse-specific plasticity takes place.

Several aspects of the relationship between NREM oscillations and learning remain largely unexplored. First, other studies have reported reactivation of neuronal ensembles during and not prior to spindles (e.g. [145]). These reactivations were observed in the motor cortex after training on a motor task, and similar increases in spindles have been reported in humans $[22,53,55]$. As discussed above, it is possible that NREM dynamics of oscillatory coupling differ between mPFC and other brain areas. Further investigations are required to address the similarities and differences between spindles and single-cell activity in different thalamocortical networks, brain regions and learning tasks. Another interesting aspect of SWR-spindle coupling is that their temporal relationship spans orders of magnitude in timescales, with SWRs preceding not only individual spindle events (at least in the mPFC) but also NREM bouts of elevated spindle event occurrence (at a timescale of approx. $10 \mathrm{~s}$ ) [93,128]. The exact role of this slow temporal coordination remains unknown. One possibility is that successive SWRs convey related messages, as observed during awake replay events [146], and thus need orchestration at a slower timescale for coherent consolidation of extended memories.

\section{(b) Spindles, a window for plasticity?}

The occurrence of spindles following SWRs may be particularly beneficial for triggering synaptic plasticity consolidation in reactivated networks (figure $5 b$ ). However, processing inputs while the network is undergoing plasticity could interfere with the consolidation of the memory trace. Interestingly, evidence suggests that the brain becomes more unresponsive to external stimuli during spindles. Combined EEG and fMRI measures in humans have shown that acoustic stimulation during spindles fails to evoke a brain response similar to the one observed outside spindles during NREM sleep $[147,148]$. In mice, arousal threshold to auditory stimuli is higher during periods of high sigma power [149]. Electrophysiological recordings in the MPFC in rats also show disengagement of cortical neuronal activity from hippocampal inputs during spindles [93] suggesting that, in addition to the external inputs, the cortex is also disconnected from some intrinsic signals. However, nociceptive signals are still transmitted to the cortex [150], which may be explained for obvious survival needs. Furthermore, contrary to what human studies predicted, neurons of the primary auditory cortex of rats respond in a similar way to auditory tone during and outside spindles [151] although it is unclear if all cortical neurons are responsive. Indeed, inhibitory control of neuronal firing during spindles is maximal in L5 pyramidal cells and not necessarily so much in superficial pyramidal neurons $[92,93]$. Overall, the apparent disengagement of cortical networks, especially of deep cortical circuits, to incoming stimuli during spindles sets a unique time window during which cortical neurons and networks may favour consolidation, a process that would otherwise not be optimal due to ongoing interference.

As described in §3a, convergent electrophysiological and imaging data suggest that $\mathrm{Ca}^{2+}$ concentration increases in dendrites in response to thalamocortical bursting activity during spindles without leading to increased firing, especially in L5 pyramidal cells. This decoupling between dendrites and 
(a)

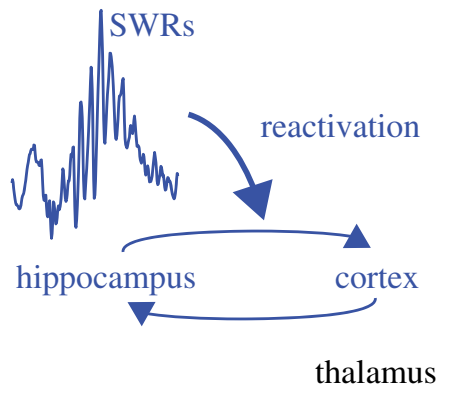

spindles

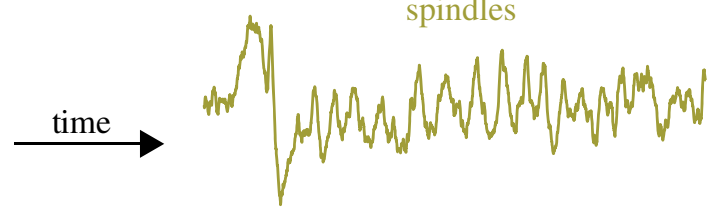

hippocampus
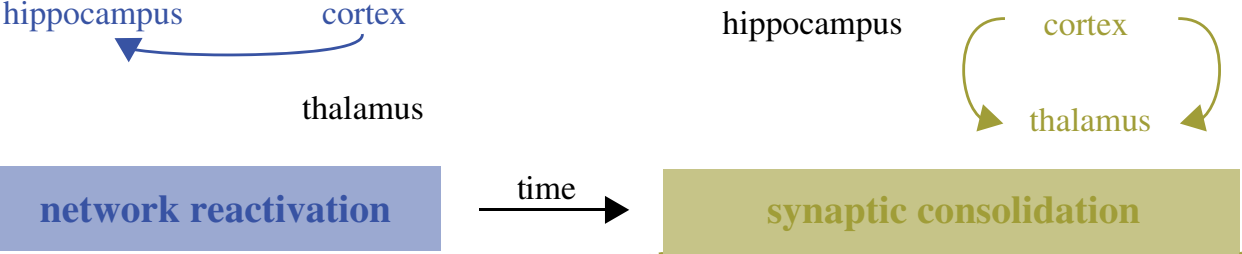

(b)

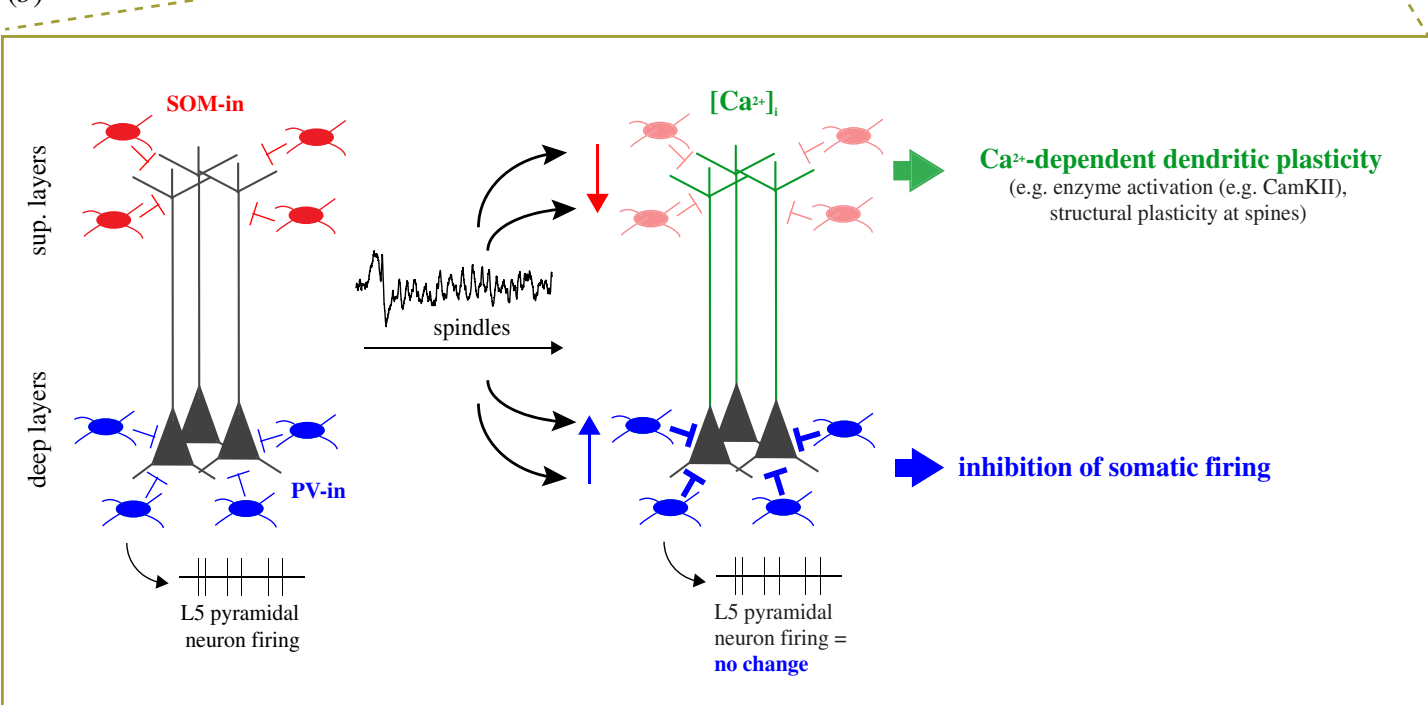

Figure 5. The sequential hypothesis of hippocampo-cortical coupling and plasticity role of spindles. (a) Illustration of the sequential hypothesis of hippocampocortical coupling. Reactivation and hippocampo-cortical network interactions during SWRs are followed by spindle events during which synaptic communication in those networks are modified via synaptic plasticity mechanisms. (b) Model of cortical circuit activity regulation of deep layers pyramidal neurons during spindles based on $\mathrm{Ca}^{2+}$ imaging, electrophysiological and computational data. During spindles, dendrites are disinhibited via decreased activity of somatostatin inhibitory (SOM-In) neurons while somatic spiking is inhibited via increase activity of parvalbumin inhibitory (PV-In) neurons. This would lead to $\mathrm{Ca}^{2+}$-dependent plasticity processes (e.g. CaMKII activation) occurring specifically in dendrites.

somatic activity most likely results from shunting inhibition by $\mathrm{PV}+$ neurons and is crucial for memory. An important role for the activity of cortical PV+ neurons for memory consolidation is supported by the observation that chemogenetic inhibition of PV+ neurons in the mPFC during NREM sleep impairs memory consolidation in mice [152]. This compartmentalization of increased intracellular $\mathrm{Ca}^{2+}$ in dendrites suggests that spindles may promote non-Hebbian type of plasticity $[153,154]$ which is accompanied by localized plasticity induction within dendrites. A variety of downstream targets and processes can be activated locally in dendrites [155] such as enzymes (e.g. CaMKII), cytoskeleton remodelling (e.g. actin dynamics) and de novo protein synthesis [19] (figure $3 d$ ). It was suggested that the frequency of $\mathrm{Ca}^{2+}$ influx could favour plasticity processes [49]. Here, CaMKII might be a particularly good candidate as its activation is sensitive to rhythmic $\mathrm{Ca}^{2+}$ influx [156,157], which may be favoured during NREM oscillations, including spindles. The type and directionality of plasticity (e.g. LTP/ LTD, functional/structural plasticity) promoted during spindles is unclear but spindles have been associated with mechanisms consistent with both potentiation and depression $[31,40,84,158]$ which may depend on their coupling with SO or Delta oscillations [84]. Future experiments using selective manipulation of thalamocortical oscillations during NREM sleep $[45,84]$ combined with cellular and molecular measures of plasticity will help address this question.

\section{Discussion}

Brain oscillations during NREM sleep have now been studied for nearly a century but it is only in recent decades that they have been related to specific neuronal activity and memory consolidation. The three hallmarks of NREM sleep, namely SWs, spindles and SWRs, are all associated with memory enhancement and plasticity. Their coordination is now well established and is thought to be at the basis for the consolidation of memories engaging distributed neuronal assemblies.

Spindles are a particularly interesting candidate for the mechanisms supporting memory consolidation in the neocortex. Here, we have reviewed how, at the systems level, their frequency of occurrence and other intrinsic features are associated with memory and how they are coupled with hippocampal replay, another key player in memory formation. Furthermore, at the cellular level, spindles regulate $\mathrm{Ca}^{2+}$ influx in neocortical dendrites, a necessary step to trigger 
mechanisms associated with plasticity. While increased dendritic activity should also lead to massive increase in neuronal spiking, studies in L5 pyramidal cells (which are the main output channel of the neocortex) show that activity in those neurons remains seemingly undisturbed by spindles, certainly controlled by shunting inhibition. This particular regulation of cortical neuron activity would enable nonHebbian plasticity processes to occur locally in dendrites/ synapses while preventing interference with ongoing activity in the circuit. The role of dendritic electrogenesis in learning has received much attention recently [159-162], and it is interesting to speculate that some of these mechanisms may be promoted during spindles as well.

In the more specific case of hippocampal-dependent memories, mPFC spindles tend to be preceded by hippocampal SWRs which are known to convey messages related to previous waking experience. This sequential ordering of SWRs and neocortical spindles may thus constitute a mechanism whereby memories are first reactivated and then consolidated.

Interestingly, the hippocampal area that is the most modulated by spindles is the dentate gyrus [133], a key structure for memory $[163,164]$. The relationship between activity in the dentate gyrus and spindles remains largely unexplored but may constitute a crucial component of sleep-dependent memory consolidation.

Although our knowledge of the dynamical processes at play for memory consolidation during sleep has made remarkable progress, many questions remain unanswered. In particular, a definitive demonstration of the link between plasticity processes, replay and SWs/spindles is still lacking. The development of precise imaging tools combined with advanced molecular approaches in behaving animals is very promising. Another question that will need to be addressed is the nature of the neuronal signal transmitted from the thalamus to the cortex. It has been assumed so far that dendrites are depolarized by excitatory volleys from the thalamus, yet, it is unlikely that this excitation is random and, instead, certainly convey meaningful messages. Recent evidence suggests that the pairwise coordination of thalamic neurons is preserved in NREM and REM sleep relative to the awake state $[165,166]$ suggesting that the cortex receives similar signals independently of brain states.

Much work on spindles still needs to be carried out. This research will not only address some fundamental questions about the role of sleep but will also open new avenues for clinical research. In fact, spindles may play a protective role in the cortex in some forms of epilepsy [167]. Furthermore, spindles are diminished in schizophrenia patients [168]) and their global coordination is impaired in mouse models of schizophrenia [129]. More generally, the covariance of spindles characteristics with neurodevelopmental and neurodegenerative disorders offers a promising use of spindles as biomarker in neuropathology. Determining a common function to spindles (and potential differences), across species, brain modalities and developmental stages $[68,169]$ is certainly one of the great challenges in sleep research in the next decade.

Data accessibility. This article has no additional data.

Authors' contributions. A.P. and J.S. wrote the manuscript. Competing interests. We have no competing interests.

Funding. This work was supported by a Canadian Research Chair in Systems Neuroscience grant no. (245716), a CIHR Project grant no. (155957), a NSERC Discovery grant no. (RGPIN-2018-04600), an IRDC grant no. (108877-001) to A.P., and the University of Surrey/ Braithwaites foundation and the Wellcome Trust grant no. (209099/ $\mathrm{Z} / 17 / \mathrm{Z}$ ) to J.S

Acknowledgements. The authors thank Adrian Duszkiewicz for comments on the manuscript.

\section{References}

1. Berger H. 1929 Über das Elektrenkephalogramm des Menschen. Arch. Für Psychiatr. Nervenkrankh. 87, 527-570. (doi:10.1007/BF01797193)

2. Loomis AL, Harvey EN, Hobart G. 1935 Potential rhythms of the cerebral cortex during sleep. Science 81, 597-598. (doi:10.1126/science.81. 2111.597)

3. Coppieters 't Wallant D, Maquet P, Phillips C. 2016 Sleep spindles as an electrographic element: description and automatic detection methods. Neural Plast. 2016, 6783812. (doi:10.1155/2016/ 6783812)

4. De Gennaro L, Ferrara M. 2003 Sleep spindles: an overview. Sleep Med. Rev. 7, $423-440$ (doi:10.1053/ smrv.2002.0252).

5. Fernandez LMJ, Luthi A. 2019 Sleep spindles: mechanisms and functions. Physiol. Rev. (doi:10. 1152/physrev.00042.2018)

6. Hobson JA, Pace-Schott EF. 2002 The cognitive neuroscience of sleep: neuronal systems, consciousness and learning. Nat. Rev. Neurosci. 3, 679-693. (doi:10.1038/nrn915)

7. Lüthi A. 2014 Sleep spindles: where they come from, what they do. Neurosci. Rev. J. Bringing
Neurobiol. Neurol. Psychiatry 20, 243-256. (doi:10. 1177/1073858413500854)

8. Astori S, Wimmer RD, Lüthi A. 2013 Manipulating sleep spindles-expanding views on sleep, memory, and disease. Trends Neurosci. 36, 738-748. (doi:10. 1016/j.tins.2013.10.001)

9. Steriade M, McCormick DA, Sejnowski TJ. 1993 Thalamocortical oscillations in the sleeping and aroused brain. Science 262, 679-685. (doi:10.1126/ science.8235588)

10. von Krosigk M, Bal T, McCormick DA. 1993 Cellular mechanisms of a synchronized oscillation in the thalamus. Science 261, 361-364. (doi:10.1126/ science.8392750)

11. Jenkins JG, Dallenbach KM. 1924 Obliviscence during sleep and waking. Am. J. Psychol. 35, 605-612. (doi:10.2307/1414040)

12. Maquet P. 2001 The role of sleep in learning and memory. Science 294, 1048-1052. (doi:10.1126/ science.1062856)

13. Navarro-Lobato I, Genzel L. 2018 The up and down of sleep: from molecules to electrophysiology. Neurobiol. Learn. Mem. 160, 3-10. (doi:10.1016/j. nIm.2018.03.013) 
20. Tononi G, Cirelli C. 2014 Sleep and the price of plasticity: from synaptic and cellular homeostasis to memory consolidation and integration. Neuron 81, 12-34. (doi:10.1016/j.neuron.2013.12.025)

21. Marshall L, Helgadóttir H, Mölle M, Born J. 2006 Boosting slow oscillations during sleep potentiates memory. Nature 444, 610-613. (doi:10.1038/ nature05278)

22. Fogel SM, Smith CT. 2006 Learning-dependent changes in sleep spindles and Stage 2 sleep. J. Sleep Res. 15, 250-255. (doi:10.1111/j.13652869.2006.00522.x)

23. Schabus M, Gruber G, Parapatics S, Sauter C, Klösch G, Anderer P, Klimesch W, Saletu B, Zeitlhofer J. 2004 Sleep spindles and their significance for declarative memory consolidation. Sleep $\mathbf{2 7}$ 1479-1485. (doi:10.1093/sleep/27.7.1479)

24. Ulrich D. 2016 Sleep spindles as facilitators of memory formation and learning. Neural Plast. 2016, 1796715. (doi:10.1155/2016/1796715)

25. Buzsáki G. 1989 Two-stage model of memory trace formation: a role for 'noisy' brain states. Neuroscience 31, 551-570. (doi:10.1016/03064522(89)90423-5)

26. Pavlides C, Winson J. 1989 Influences of hippocampal place cell firing in the awake state on the activity of these cells during subsequent sleep episodes. J. Neurosci. Off. J. Soc. Neurosci. 9, 2907-2918 (doi:10.1523/JNEUROSCI.09-08-02907. 1989).

27. Wilson MA, McNaughton BL. 1994 Reactivation of hippocampal ensemble memories during sleep. Science 265, 676-679. (doi:10.1126/science. 8036517)

28. Genzel L, Kroes MCW, Dresler M, Battaglia FP. 2014 Light sleep versus slow wave sleep in memory consolidation: a question of global versus local processes? Trends Neurosci. 37, 10-19. (doi:10. 1016/j.tins.2013.10.002)

29. Dijk DJ, Hayes B, Czeisler CA. 1993 Dynamics of electroencephalographic sleep spindles and slow wave activity in men: effect of sleep deprivation. Brain Res. 626, 190-199 (doi:10.1016/00068993(93)90579-C).

30. Gottesmann C. 1996 The transition from slow-wave sleep to paradoxical sleep: evolving facts and concepts of the neurophysiological processes underlying the intermediate stage of sleep. Neurosci. Biobehav. Rev. 20, 367-387 (doi:10.1016/ 0149-7634(95)00055-0).

31. Seibt J, Richard CJ, Sigl-Glöckner J, Takahashi N, Kaplan DI, Doron G, de Limoges D, Bocklisch C, Larkum ME. 2017 Cortical dendritic activity correlates with spindle-rich oscillations during sleep in rodents. Nat. Commun. 8, 684. (doi:10.1038/ s41467-017-00735-w)

32. Watson BO, Levenstein D, Greene JP, Gelinas JN, Buzsáki G. 2016 Network homeostasis and state dynamics of neocortical sleep. Neuron 90, 839-852. (doi:10.1016/j.neuron.2016.03.036)

33. Clawson BC, Durkin J, Aton SJ. 2016 Form and function of sleep spindles across the lifespan. Neural Plast. 2016, 6936381. (doi:10.1155/2016/6936381)
34. Crunelli V, Lőrincz ML, Connelly WM, David F, Hughes SW, Lambert RC, Leresche N, Errington AC. 2018 Dual function of thalamic low-vigilance state oscillations: rhythm-regulation and plasticity. Nat. Rev. Neurosci. 19, 107-118. (doi:10.1038/nrn.2017. 151)

35. Crunelli V, Hughes SW. 2010 The slow $(<1 \mathrm{~Hz})$ rhythm of non-REM sleep: a dialogue between three cardinal oscillators. Nat. Neurosci. 13, 9-17. (doi:10.1038/nn.2445)

36. McCormick DA, Bal T. 1997 Sleep and arousal: thalamocortical mechanisms. Annu. Rev. Neurosci. 20, 185-215. (doi:10.1146/annurev.neuro.20.1.185)

37. Dehghani N, Cash SS, Rossetti A0, Chen CC, Halgren E. 2010 Magnetoencephalography demonstrates multiple asynchronous generators during human sleep spindles. J. Neurophysiol. 104, 179-188. (doi:10.1152/jn.00198.2010)

38. Gais S, Mölle M, Helms K, Born J. 2002 Learningdependent increases in sleep spindle density. J. Neurosci. Off. J. Soc. Neurosci. 22, 6830-6834. (doi:20026697).

39. Eschenko 0, Mölle M, Born J, Sara SJ. 2006 Elevated sleep spindle density after learning or after retrieval in rats. J. Neurosci. Off. J. Soc. Neurosci. 26, 12 914-12 920. (doi:10.1523/JNEUROSCI.3175-06.2006)

40. Rosanova M, Ulrich D. 2005 Pattern-specific associative long-term potentiation induced by a sleep spindle-related spike train. J. Neurosci. Off. J. Soc. Neurosci. 25, 9398-9405. (doi:10.1523/ JNEUROSCI.2149-05.2005)

41. Contreras D, Destexhe A, Sejnowski TJ, Steriade M. 1996 Control of spatiotemporal coherence of a thalamic oscillation by corticothalamic feedback. Science 274, 771-774. (doi:10.1126/science.274. 5288.771)

42. Buzsáki G, Logothetis N, Singer W. 2013 Scaling brain size, keeping timing: evolutionary preservation of brain rhythms. Neuron 80, 751-764. (doi:10. 1016/j.neuron.2013.10.002)

43. Andrillon T, Nir Y, Staba RJ, Ferrarelli F, Cirelli C, Tononi G, Fried I. 2011 Sleep spindles in humans: insights from intracranial EEG and unit recordings. J. Neurosci. Off. J. Soc. Neurosci. 31, 17 821-17 834. (doi:10.1523/JNEUROSCI.2604-11.2011)

44. Mölle M, Eschenko 0, Gais S, Sara SJ, Born J. 2009 The influence of learning on sleep slow oscillations and associated spindles and ripples in humans and rats. Eur. J. Neurosci. 29, 1071-1081. (doi:10.1111/ j.1460-9568.2009.06654.x)

45. Fernandez LM, Vantomme G, Osorio-Forero A, Cardis R, Béard E, Lüthi A. 2018 Thalamic reticular control of local sleep in mouse sensory cortex. elife 7, e39111. (doi:10.7554/elife.39111)

46. Kim D, Hwang E, Lee M, Sung H, Choi JH. 2015 Characterization of topographically specific sleep spindles in mice. Sleep 38, 85-96. (doi:10.5665) sleep.4330)

47. Frauscher B, von Ellenrieder N, Dubeau F, Gotman J. 2015 Scalp spindles are associated with widespread intracranial activity with unexpectedly low synchrony. Neuroimage 105, 1-12. (doi:10.1016/j. neuroimage.2014.10.048)
48. Nir Y, Staba RJ, Andrillon T, Vyazovskiy VV, Cirelli C, Fried I, Tononi G. 2011 Regional slow waves and spindles in human sleep. Neuron 70, 153-169. (doi:10.1016/j.neuron.2011.02.043)

49. Sejnowski TJ, Destexhe A. 2000 Why do we sleep? Brain Res. 886, 208-223 (doi:10.1016/500068993(00)03007-9).

50. Steriade M, Timofeev I. 2003 Neuronal plasticity in thalamocortical networks during sleep and waking oscillations. Neuron 37, 563-576 (doi:10.1016/ S0896-6273(03)00065-5).

51. Barakat $M$ et al. 2013 Sleep spindles predict neura and behavioral changes in motor sequence consolidation. Hum. Brain Mapp. 34, 2918-2928. (doi:10.1002/hbm.22116)

52. Clemens Z, Fabó D, Halász P. 2005 Overnight verbal memory retention correlates with the number of sleep spindles. Neuroscience 132, 529-535. (doi:10. 1016/j.neuroscience.2005.01.011)

53. Laventure $S$ et al. 2016 NREM2 and sleep spindles are instrumental to the consolidation of motor sequence memories. PLOS Biol. 14, e1002429. (doi:10.1371/journal.pbio.1002429)

54. Meier-Koll A, Bussmann B, Schmidt C, Neuschwander D. 1999 Walking through a maze alters the architecture of sleep. Percept. Mot. Skills 88, 1141-1159. (doi:10.2466/pms.1999.88.3c.1141)

55. Nishida M, Walker MP. 2007 Daytime naps, motor memory consolidation and regionally specific sleep spindles. PLOS ONE 2, e341. (doi:10.1371/journal. pone.0000341)

56. Ackermann S, Hartmann F, Papassotiropoulos A, de Quervain DJ-F, Rasch B. 2015 No associations between interindividual differences in sleep parameters and episodic memory consolidation. Sleep 38, 951-959. (doi:10.5665/sleep.4748)

57. Bergmann T0, Mölle M, Diedrichs J, Born J, Siebner HR. 2012 Sleep spindle-related reactivation of category-specific cortical regions after learning facescene associations. Neuroimage 59, 2733-2742. (doi:10.1016/j.neuroimage.2011.10.036)

58. Cox R, Hofman WF, de Boer M, Talamini LM. 2014 Local sleep spindle modulations in relation to specific memory cues. Neuroimage 99, 103-110. (doi:10.1016/j.neuroimage.2014.05.028)

59. Jegou A et al. 2019 Cortical reactivations during sleep spindles following declarative learning. Neuroimage 195, 104-112. (doi:10.1016/j. neuroimage.2019.03.051)

60. Johnson LA, Blakely T, Hermes D, Hakimian S, Ramsey NF, Ojemann JG. 2012 Sleep spindles are locally modulated by training on a brain-computer interface. Proc. Natl Acad. Sci. USA 109, 18583-18 588. (doi:10.1073/pnas.1207532109)

61. Huber R, Ghilardi MF, Massimini M, Ferrarelli F, Riedner BA, Peterson MJ, Tononi G. 2006 Arm immobilization causes cortical plastic changes and locally decreases sleep slow wave activity. Nat. Neurosci. 9, 1169-1176. (doi:10.1038/ $\mathrm{nn} 1758)$

62. Huber R, Ghilardi MF, Massimini M, Tononi G. 2004 Local sleep and learning. Nature 430, 78-81. (doi:10.1038/nature02663) 
63. Durkin J, Suresh AK, Colbath J, Broussard C, Wu J, Zochowski M, Aton SJ. 2017 Cortically coordinated NREM thalamocortical oscillations play an essential, instructive role in visual system plasticity. Proc. Natl Acad. Sci. USA 114, 10 485-10 490. (doi:10.1073/ pnas.1710613114)

64. Vyazovskiy V, Borbély AA, Tobler I. 2000 Unilateral vibrissae stimulation during waking induces interhemispheric EEG asymmetry during subsequent sleep in the rat. J. Sleep Res. 9, 367-371 (doi:10. 1046/j.1365-2869.2000.00230.x).

65. Aton SJ, Suresh A, Broussard C, Frank MG. 2014 Sleep promotes cortical response potentiation following visual experience. Sleep 37, 1163-1170. (doi:10.5665/sleep.3830)

66. Aton SJ, Broussard C, Dumoulin M, Seibt J, Watson A, Coleman T, Frank MG. 2013 Visual experience and subsequent sleep induce sequential plastic changes in putative inhibitory and excitatory cortical neurons. Proc. Natl Acad. Sci. USA 110, 3101-3106. (doi:10.1073/pnas.1208093110)

67. Hanganu IL, Ben-Ari Y, Khazipov R. 2006 Retinal waves trigger spindle bursts in the neonatal rat visual cortex. J. Neurosci. Off. J. Soc. Neurosci. 26, 6728-6736. (doi:10.1523/JNEUROSCI.0752-06.2006)

68. Khazipov R, Sirota A, Leinekugel X, Holmes GL, BenAri Y, Buzsáki G. 2004 Early motor activity drives spindle bursts in the developing somatosensory cortex. Nature 432, 758-761. (doi:10.1038/ nature03132)

69. Lindemann C, Ahlbeck J, Bitzenhofer SH, HanganuOpatz IL. 2016 Spindle activity orchestrates plasticity during development and sleep. Neural Plast. 2016, 5787423. (doi:10.1155/2016/5787423)

70. Gruber R, Wise MS. 2016 Sleep spindle characteristics in children with neurodevelopmental disorders and their relation to cognition. Neural Plast. 2016, 4724792. (doi:10.1155/2016/4724792)

71. Manoach DS, Pan JQ, Purcell SM, Stickgold R. 2016 Reduced sleep spindles in schizophrenia: a treatable endophenotype that links risk genes to impaired cognition? Biol. Psychiatry 80, 599-608. (doi:10. 1016/j.biopsych.2015.10.003)

72. Winsky-Sommerer R, de Oliveira $P$, Loomis $S$, Wafford K, Dijk D-J, Gilmour G. 2018 Disturbances of sleep quality, timing and structure and their relationship with other neuropsychiatric symptoms in Alzheimer's disease and schizophrenia: insights from studies in patient populations and animal models. Neurosci. Biobehav. Rev. 97, 112-137. (doi:10.1016/j.neubiorev. 2018.09.027)

73. Cellini N, Mednick SC. 2019 Stimulating the sleeping brain: current approaches to modulating memory-related sleep physiology. J. Neurosci. Methods 316, 125-136. (doi:10.1016/j.jneumeth. 2018.11.011)

74. Lustenberger C, Boyle MR, Alagapan S, Mellin JM, Vaughn BV, Fröhlich F. 2016 Feedback-controlled transcranial alternating current stimulation reveals a functional role of sleep spindles in motor memory consolidation. Curr. Biol. CB 26, 2127-2136. (doi:10.1016/j.cub.2016.06.044)
75. Niknazar M, Krishnan GP, Bazhenov M, Mednick SC. 2015 Coupling of thalamocortical sleep oscillations are important for memory consolidation in humans. PLOS ONE 10, e0144720. (doi:10.1371/journal.pone. 0144720)

76. Rasch B, Büchel C, Gais S, Born J. 2007 Odor cues during slow-wave sleep prompt declarative memory consolidation. Science 315, 1426-1429. (doi:10. 1126/science.1138581)

77. Rudoy JD, Voss JL, Westerberg CE, Paller KA. 2009 Strengthening individual memories by reactivating them during sleep. Science 326, 1079. (doi:10.1126/ science.1179013)

78. Cairney SA, Guttesen AÁV, El Marj N, Staresina BP. 2018 Memory consolidation is linked to spindlemediated information processing during sleep. Curr. Biol. CB 28, 948-954. e4. (doi:10.1016/j.cub.2018. 01.087.)

79. Rihm JS, Diekelmann S, Born J, Rasch B. 2014 Reactivating memories during sleep by odors: odor specificity and associated changes in sleep oscillations. J. Cogn. Neurosci. 26, 1806-1818. (doi:10.1162/jocn_a_00579)

80. Perrault AA, Khani A, Quairiaux C, Kompotis K, Franken $P$, Muhlethaler M, Schwartz S, Bayer L. 2019 Whole-night continuous rocking entrains spontaneous neural oscillations with benefits for sleep and memory. Curr. Biol. 29, 402-411.e3. (doi:10.1016/j.cub.2018.12.028)

81. Kompotis K, Hubbard J, Emmenegger Y, Perrault A, Mühlethaler M, Schwartz S, Bayer L, Franken P. 2019 Rocking promotes sleep in mice through rhythmic stimulation of the vestibular system. Curr. Biol. 29, 392-401.e4. (doi:10.1016/j.cub.2018.12. 007)

82. Latchoumane (-FV, Ngo H-VV, Born J, Shin H-S. 2017 Thalamic spindles promote memory formation during sleep through triple phase-locking of cortical, thalamic, and hippocampal rhythms. Neuron 95, 424-435.e6. (doi:10.1016/j.neuron. 2017.06.025)

83. Kim A, Latchoumane C, Lee S, Kim GB, Cheong E, Augustine GJ, Shin H-S. 2012 Optogenetically induced sleep spindle rhythms alter sleep architectures in mice. Proc. Natl Acad. Sci. USA 109, 20 673-20 678. (doi:10.1073/pnas.1217897109)

84. Kim J, Gulati T, Ganguly K. 2019 Competing roles of slow oscillations and delta waves in memory consolidation versus forgetting. Cell 179, 514-526.e13. (doi:10.1016/j.cell.2019.08.040)

85. Bear MF, Abraham WC. 1996 Long-term depression in hippocampus. Annu. Rev. Neurosci. 19, 437-462. (doi:10.1146/annurev.ne.19.030196.002253)

86. Malenka RC, Nicoll RA. 1999 Long-term potentiation-a decade of progress? Science 285, 1870-1874. (doi:10.1126/science.285.5435.1870)

87. Nabavi S, Fox R, Proulx CD, Lin JY, Tsien RY, Malinow R. 2014 Engineering a memory with LTD and LTP. Nature 511, 348-352. (doi:10.1038/ nature13294)

88. Takeuchi T, Duszkiewicz AJ, Morris RGM. 2014 The synaptic plasticity and memory hypothesis: encoding, storage and persistence. Phil.
Trans. R. Soc. B 369, 20130288. (doi:10.1098/rstb. 2013.0288)

89. Whitlock JR, Heynen AJ, Shuler MG, Bear MF. 2006 Learning induces long-term potentiation in the hippocampus. Science 313, 1093-1097. (doi:10. 1126/science.1128134)

90. Bergmann TO, Mölle M, Marshall L, Kaya-Yildiz L, Born J, Roman Siebner H. 2008 A local signature of LTP- and LTD-like plasticity in human NREM sleep. Eur. J. Neurosci. 27, 2241-2249. (doi:10.1111/j. 1460-9568.2008.06178.x)

91. Chauvette S, Seigneur J, Timofeev I. 2012 Sleep oscillations in the thalamocortical system induce long-term neuronal plasticity. Neuron 75, 1105-1113. (doi:10.1016/j.neuron.2012.08.034)

92. Contreras D, Destexhe A, Steriade M. 1997 Intracellular and computational characterization of the intracortical inhibitory control of synchronized thalamic inputs in vivo. J. Neurophysiol. 78, 335-350 (doi:10.1152/jn.1997.78.1.335).

93. Peyrache A, Battaglia FP, Destexhe A. 2011 Inhibition recruitment in prefrontal cortex during sleep spindles and gating of hippocampal inputs. Proc. Natl Acad. Sci. USA 108, 17 207-17 212. (doi:10.1073/pnas.1103612108)

94. Blanco W et al. 2015 Synaptic homeostasis and restructuring across the sleep-wake cycle. PLoS Comput. Biol. 11, e1004241. (doi:10.1371/journal. pcbi.1004241)

95. Cruikshank SJ, Lewis TJ, Connors BW. 2007 Synaptic basis for intense thalamocortical activation of feedforward inhibitory cells in neocortex. Nat. Neurosci. 10, 462-468. (doi:10.1038/nn1861)

96. Haider B, Häusser M, Carandini M. 2013 Inhibition dominates sensory responses in awake cortex. Nature 493, 97-100. (doi:10.1038/nature11665)

97. Porter JT, Johnson CK, Agmon A. 2001 Diverse types of interneurons generate thalamus-evoked feedforward inhibition in the mouse barrel cortex. J. Neurosci. 21, 2699-2710. (doi:10.1523/ JNEUROSCI.21-08-02699.2001)

98. Swadlow HA. 2002 Thalamocortical control of feedforward inhibition in awake somatosensory 'barrel' cortex. Phil. Trans. R. Soc. B 357, 1717-1727. (doi:10.1098/rstb.2002.1156)

99. Hartwich K, Pollak T, Klausberger T. 2009 Distinct firing patterns of identified basket and dendritetargeting interneurons in the prefrontal cortex during hippocampal theta and local spindle oscillations. J. Neurosci. Off. J. Soc. Neurosci. 29, 9563-9574. (doi:10.1523/JNEUROSCI.139709.2009)

100. Constantinople CM, Bruno RM. 2013 Deep cortical layers are activated directly by thalamus. Science 340, 1591-1594. (doi:10.1126/science.1236425)

101. Niethard N, Ngo H-VV, Ehrlich I, Born J. 2018 Cortical circuit activity underlying sleep slow oscillations and spindles. Proc. Natl Acad. Sci. USA 115, E9220-E9229. (doi:10.1073/pnas.1805517115)

102. Niethard N, Burgalossi A, Born J. 2017 Plasticity during sleep is linked to specific regulation of cortical circuit activity. Front. Neural Circuits 11, 65. (doi:10.3389/fncir.2017.00065) 
103. Sigl-Glöckner J, Seibt J. 2018 Peeking into the sleeping brain: using in vivo imaging in rodents to understand the relationship between sleep and cognition. J. Neurosci. Methods 316, 71-82. (doi:10. 1016/j.jneumeth.2018.09.011)

104. Tremblay R, Lee S, Rudy B. 2016 GABAergic interneurons in the neocortex: from cellular properties to circuits. Neuron 91, 260-292. (doi:10. 1016/j.neuron.2016.06.033)

105. Kim JJ, Fanselow MS. 1992 Modality-specific retrograde amnesia of fear. Science 256, 675-677. (doi:10.1126/science.1585183)

106. McClelland JL, McNaughton BL, O'Reilly RC. 1995 Why there are complementary learning systems in the hippocampus and neocortex: insights from the successes and failures of connectionist models of learning and memory. Psychol. Rev. 102, 419-457. (doi:10.1037/0033-295X.102.3.419)

107. Nadel L, Moscovitch M. 1997 Memory consolidation, retrograde amnesia and the hippocampal complex. Curr. Opin. Neurobiol. 7, 217-227 (doi:10.1016/ S0959-4388(97)80010-4).

108. Scoville WB, Milner B. 1957 Loss of recent memory after bilateral hippocampal lesions. J. Neurol. Neurosurg. Psychiatry 20, 11-21. (doi:10.1136/jnnp. 20.1.11)

109. Squire LR, Alvarez P. 1995 Retrograde amnesia and memory consolidation: a neurobiological perspective. Curr. Opin. Neurobiol. 5, 169-177 (doi:10.1016/0959-4388(95)80023-9).

110. Buzsáki G. 2015 Hippocampal sharp wave-ripple: a cognitive biomarker for episodic memory and planning. Hippocampus 25, 1073-1188. (doi:10. 1002/hipo.22488)

111. Peyrache A, Khamassi M, Benchenane K, Wiener SI, Battaglia FP. 2009 Replay of rule-learning related neural patterns in the prefrontal cortex during sleep. Nat. Neurosci. 12, 919-926. (doi:10.1038/nn. 2337)

112. Ego-Stengel V, Wilson MA. 2010 Disruption of ripple-associated hippocampal activity during rest impairs spatial learning in the rat. Hippocampus 20, 1-10. (doi:10.1002/hipo.20707)

113. Girardeau G, Benchenane K, Wiener SI, Buzsáki G, Zugaro MB. 2009 Selective suppression of hippocampal ripples impairs spatial memory. Nat. Neurosci. 12, 1222-1223. (doi:10.1038/nn.2384)

114. Diekelmann S, Born J. 2010 The memory function of sleep. Nat. Rev. Neurosci. 11, 114-126. (doi:10. 1038/nrn2762)

115. O'Neill J, Pleydell-Bouverie B, Dupret D, Csicsvari J. 2010 Play it again: reactivation of waking experience and memory. Trends Neurosci. 33, 220-229. (doi:10.1016/j.tins.2010.01.006)

116. Tingley D, Peyrache A. 2020 On the methods for reactivation and replay analysis. Phil. Trans. R. Soc. B 375, 20190231. (doi:10.1098/rstb.2019.0231)

117. Lee AK, Wilson MA. 2002 Memory of sequential experience in the hippocampus during slow wave sleep. Neuron 36, 1183-1194 (doi:10.1016/508966273(02)01096-6).

118. Chrobak JJ, Buzsáki G. 1994 Selective activation of deep layer (V-VI) retrohippocampal cortical neurons during hippocampal sharp waves in the behaving rat. J. Neurosci. Off. J. Soc. Neurosci. 14, 6160-6170 (doi:10.1523/JNEUROSCI.14-10-06160.1994).

119. Ólafsdóttir HF, Carpenter F, Barry C. 2016 Coordinated grid and place cell replay during rest. Nat. Neurosci. 19, 792-794. (doi:10.1038/nn.4291)

120. O'Neill J, Boccara CN, Stella F, Schoenenberger $P$, Csicsvari J. 2017 Superficial layers of the medial entorhinal cortex replay independently of the hippocampus. Science 355, 184-188. (doi:10.1126/ science.aag2787)

121. Lansink CS, Goltstein PM, Lankelma JV, McNaughton BL, Pennartz CMA. 2009 Hippocampus leads ventral striatum in replay of place-reward information. PLoS Biol. 7, e1000173. (doi:10.1371/journal.pbio. 1000173)

122. Sjulson L, Peyrache A, Cumpelik A, Cassataro D, Buzsáki G. 2018 Cocaine place conditioning strengthens location-specific hippocampal coupling to the nucleus accumbens. Neuron 98, 926-934.e5. (doi:10.1016/j.neuron.2018.04.015)

123. Girardeau G, Inema I, Buzsáki G. 2017 Reactivations of emotional memory in the hippocampusamygdala system during sleep. Nat. Neurosci. 20, 1634-1642. (doi:10.1038/nn.4637)

124. Eschenko 0, Ramadan W, Mölle M, Born J, Sara SJ. 2008 Sustained increase in hippocampal sharp-wave ripple activity during slow-wave sleep after learning. Learn. Mem. 15, 222-228. (doi:10.1101/ Im.726008)

125. Girardeau G, Cei A, Zugaro M. 2014 Learninginduced plasticity regulates hippocampal sharp wave-ripple drive. J. Neurosci. Off. J. Soc. Neurosci. 34, 5176-5183. (doi:10.1523/JNEUROSCI.4288-13. 2014)

126. Norimoto $\mathrm{H}$ et al. 2018 Hippocampal ripples downregulate synapses. Science 359, 1524-1527. (doi:10. 1126/science.aa00702)

127. Frankland PW, Bontempi B. 2005 The organization of recent and remote memories. Nat. Rev. Neurosci. 6, 119-130. (doi:10.1038/nrn1607)

128. Siapas AG, Wilson MA. 1998 Coordinated interactions between hippocampal ripples and cortical spindles during slow-wave sleep. Neuron 21, 1123-1128 (doi:10.1016/50896-6273(00) 80629-7).

129. Phillips KG, Bartsch U, McCarthy AP, Edgar DM, Tricklebank MD, Wafford KA, Jones MW. 2012 Decoupling of sleep-dependent cortical and hippocampal interactions in a neurodevelopmental model of schizophrenia. Neuron 76, 526-533. (doi:10.1016/j.neuron.2012.09.016)

130. Sirota A, Csicsvari J, Buhl D, Buzsáki G. 2003 Communication between neocortex and hippocampus during sleep in rodents. Proc. Natl Acad. Sci. USA 100, 2065-2069. (doi:10.1073/pnas. 0437938100)

131. Isomura Y, Sirota A, Ozen S, Montgomery S, Mizuseki K, Henze DA, Buzsáki G. 2006 Integration and segregation of activity in entorhinalhippocampal subregions by neocortical slow oscillations. Neuron 52, 871-882. (doi:10.1016/j. neuron.2006.10.023)
132. Mölle M, Yeshenko 0, Marshall L, Sara SJ, Born J. 2006 Hippocampal sharp wave-ripples linked to slow oscillations in rat slow-wave sleep. J. Neurophysiol. 96, 62-70. (doi:10.1152/jn.00014. 2006)

133. Sullivan D, Mizuseki K, Sorgi A, Buzsáki G. 2014 Comparison of sleep spindles and theta oscillations in the hippocampus. J. Neurosci. Off. J. Soc. Neurosci. 34, 662-674. (doi:10.1523/JNEUROSCl. 0552-13.2014)

134. Varela C, Wilson MA. 2019 mPFC spindle cycles organize sparse thalamic activation and recently active ca1 cells during non-REM Sleep. bioRxiv 653436. (doi:10.1101/653436)

135. Viejo G, Peyrache A. 2019 Precise coupling of the thalamic head-direction system to hippocampal ripples. bioRxiv. (doi:10.1101/809657)

136. Yang M, Logothetis NK, Eschenko 0. 2019 Occurrence of hippocampal ripples is associated with activity suppression in the mediodorsal thalamic nucleus. J. Neurosci. Off. J. Soc. Neurosci. 39, 434-444. (doi:10.1523/JNEUROSCI.2107-18. 2018)

137. Norman $Y$, Yeagle EM, Khuvis $S$, Harel M, Mehta $A D$, Malach R. 2019 Hippocampal sharp-wave ripples linked to visual episodic recollection in humans. Science 365, eaax1030. (doi:10.1126/science. aax1030)

138. Vaz AP, Inati SK, Brunel N, Zaghloul KA. 2019 Coupled ripple oscillations between the medial temporal lobe and neocortex retrieve human memory. Science 363, 975-978. (doi:10.1126/ science.aau8956)

139. Clemens Z, Mölle M, Eross L, Jakus $R$, Rásonyi $G$, Halász P, Born J. 2011 Fine-tuned coupling between human parahippocampal ripples and sleep spindles. Eur. J. Neurosci. 33, 511-520. (doi:10.1111/j.14609568.2010.07505.x)

140. Staresina BP, Bergmann T0, Bonnefond M, van der Meij $\mathrm{R}$, Jensen 0, Deuker L, Elger CE, Axmacher N, Fell J. 2015 Hierarchical nesting of slow oscillations, spindles and ripples in the human hippocampus during sleep. Nat. Neurosci. 18, 1679-1686. (doi:10. 1038/nn.4119)

141. Maingret, coll. 2016.

142. Maingret N, Girardeau G, Todorova R, Goutierre M, Zugaro M. 2016 Hippocampo-cortical coupling mediates memory consolidation during sleep. Nat. Neurosci. 19, 959-964. (doi:10.1038/ nn.4304)

143. Jay TM, Glowinski J, Thierry AM. 1989 Selectivity of the hippocampal projection to the prelimbic area of the prefrontal cortex in the rat. Brain Res. 505, 337-340. (doi:10.1016/0006-8993(89) 91464-9)

144. Maviel T, Durkin TP, Menzaghi F, Bontempi B. 2004 Sites of neocortical reorganization critical for remote spatial memory. Science 305, 96-99. (doi:10.1126/ science.1098180)

145. Ramanathan DS, Gulati T, Ganguly K. 2015 Sleepdependent reactivation of ensembles in motor cortex promotes skill consolidation. PLOS Biol. 13, e1002263. (doi:10.1371/journal.pbio.1002263) 
146. Davidson TJ, Kloosterman F, Wilson MA. 2009 Hippocampal replay of extended experience. Neuron 63, 497-507. (doi:10.1016/j.neuron.2009.07.027)

147. Dang-Vu TT et al. 2011 Interplay between spontaneous and induced brain activity during human non-rapid eye movement sleep. Proc. Natl Acad. Sci. USA 108, 15 438-15 443. (doi:10.1073/ pnas.1112503108)

148. Schabus $\mathrm{M}$ et al. 2012 The fate of incoming stimuli during NREM sleep is determined by spindles and the phase of the slow oscillation. Front. Neurol. 3, 40. (doi:10.3389/fneur.2012.00040)

149. Lecci S, Fernandez LMJ, Weber FD, Cardis $R$, Chatton J-Y, Born J, Lüthi A. 2017 Coordinated infraslow neural and cardiac oscillations mark fragility and offline periods in mammalian sleep. Sci. Adv. 3, e1602026. (doi:10.1126/sciadv.1602026)

150. Claude L, Chouchou F, Prados G, Castro M, De Blay B, Perchet C, García-Larrea L, Mazza S, Bastuji H. 2015 Sleep spindles and human cortical nociception: a surface and intracerebral electrophysiological study. J. Physiol. 593, 4995-5008. (doi:10.1113/JP270941)

151. Sela Y, Vyazovskiy VV, Cirelli C, Tononi G, Nir Y. 2016 Responses in rat core auditory cortex are preserved during sleep spindle oscillations. Sleep $\mathbf{3 9}$, 1069-1082. (doi:10.5665/sleep.5758)

152. Xia F, Richards BA, Tran MM, Josselyn SA, TakeharaNishiuchi K, Frankland PW. 2017 Parvalbuminpositive interneurons mediate neocorticalhippocampal interactions that are necessary for memory consolidation. elife 6, e27868. (doi:10. 7554/eLife.27868)

153. Gambino F, Pagès $S$, Kehayas V, Baptista $D$, Tatti $R$, Carleton A, Holtmaat A. 2014 Sensory-evoked
LTP driven by dendritic plateau potentials in vivo. Nature 515, 116-119. (doi:10.1038/ nature13664)

154. Golding NL, Staff NP, Spruston N. 2002 Dendritic spikes as a mechanism for cooperative long-term potentiation. Nature 418, 326-331. (doi:10.1038/ nature00854)

155. Ho VM, Lee J-A, Martin KC. 2011 The cell biology of synaptic plasticity. Science 334, 623-628. (doi:10. 1126/science. 1209236)

156. Dupont G, Goldbeter A. 1998 CaM kinase II as frequency decoder of Ca2+ oscillations. BioEssays News Rev. Mol. Cell. Dev. Biol. 20, 607-610. (doi:10. 1002/(SICI)1521-1878(199808)20:8<607::AIDBIES2>3.0.(0;2-F)

157. Koninck PD, Schulman H. 1998 Sensitivity of CaM Kinase II to the frequency of $\mathrm{Ca} 2+$ oscillations. Science 279, 227-230. (doi:10.1126/science.279. 5348.227)

158. Miyawaki H, Diba K. 2016 Regulation of hippocampal firing by network oscillations during sleep. Curr. Biol. CB 26, 893-902. (doi:10.1016/j. cub.2016.02.024)

159. Bittner KC, Milstein AD, Grienberger C, Romani S, Magee JC. 2017 Behavioral time scale synaptic plasticity underlies CA1 place fields. Science 357, 1033-1036. (doi:10.1126/science.aan3846)

160. Guerguiev J, Lillicrap TP, Richards BA. 2017 Towards deep learning with segregated dendrites. elife 6 . (doi:10.7554/elife.22901)

161. Kastellakis G, Cai DJ, Mednick SC, Silva AJ, Poirazi P. 2015 Synaptic clustering within dendrites: an emerging theory of memory formation. Prog. Neurobiol. 126, 19-35. (doi:10.1016/j.pneurobio. 2014.12.002)
162. Mel BW, Schiller J, Poirazi P. 2017 Synaptic plasticity in dendrites: complications and coping strategies. Curr. Opin. Neurobiol. 43, 177-186. (doi:10.1016/j. conb.2017.03.012)

163. Kesner RP. 2018 An analysis of dentate gyrus function (an update). Behav. Brain Res. 354, 84-91. (doi:10.1016/j.bbr.2017.07.033)

164. Senzai Y. 2019 Function of local circuits in the hippocampal dentate gyrus-CA3 system. Neurosci. Res. 140, 43-52. (doi:10.1016/..neures.2018.11. 003)

165. Chaudhuri R, Gerçek B, Pandey B, Peyrache A, Fiete I. 2019 The intrinsic attractor manifold and population dynamics of a canonical cognitive circuit across waking and sleep. Nat. Neurosci. 22, 1512-1520. (doi:10.1038/s41593-0190460-x)

166. Peyrache A, Lacroix MM, Petersen PC, Buzsáki G. 2015 Internally organized mechanisms of the head direction sense. Nat. Neurosci. 18, 569-575. (doi:10. 1038/nn.3968)

167. Gelinas JN, Khodagholy D, Thesen T, Devinsky 0, Buzsáki G. 2016 Interictal epileptiform discharges induce hippocampal-cortical coupling in temporal lobe epilepsy. Nat. Med. 22, 641-648. (doi:10.1038/ nm.4084)

168. Ferrarelli F, Huber R, Peterson MJ, Massimini $M$, Murphy M, Riedner BA, Watson A, Bria P, Tononi G. 2007 Reduced sleep spindle activity in schizophrenia patients. Am. J. Psychiatry 164, 483-492. (doi:10. 1176/appi.ajp.164.3.483)

169. Khazipov R, Luhmann HJ. 2006 Early patterns of electrical activity in the developing cerebral cortex of humans and rodents. Trends Neurosci. 29, 414-418. (doi:10.1016/j.tins.2006.05.007) 